\title{
PSYCHE
}

\begin{tabular}{lll}
\hline VOL. XXVIII JUNE, 1921 & No. 3
\end{tabular}

\section{MUSCULATURE AND MECHANISM OF MOVEMENT OF THE TARSI IN APHIDS. ${ }^{1}$}

By Leopoldo B. Uichanco, College of Agriculture, University of the Philippines, Los Baños, P. I.

The present work is an attempt to determine the muscles responsible for the movement of the tarsi in aphids. The manner of articulation of the first tarsal segment on the tibia and the mechanism of movement of the tarsi are discussed in some detail.

\section{Material and Methods.}

The form used was Myzus persica Sulzer. Like many other aphids, this species is a very favorable subject for anatomical study of the appendages on account of the semi-transparent condition of the integument, which renders the interior more or less visible, even in the living specimen. Two series were studied: (1) living material and (2) balsam preparations in toto. For the study of the living insects, both nymphs and adults, mounted in aqueous or saline media, were used. In these it was possible to follow the movements of the tarsi under both the 16-millimeter and the 4-millimeter objectives of an ordinary Bausch and Lomb microscope fitted with a No. 10 ocular. The muscle responsible for the movement and its tendons also showed a fairly sharp definition in the fresh, unstained preparations. Amputated legs were likewise studied, although not used as a basis for the investigation of tarsal movements for the reason that the operation might have brought about conditions which would tend to produce abnormal reflexes. A more careful preliminary anatomical study was made of fresil specimens with the aid of Schneider's acid carmine. This reagent was found to stain the nuclei of the hypodermal cells and the muscle bodies deeply, differentiating them fairly well from the

1 Contributions from the Entomological Laboratories of the Bussey Institution for Research in Applied Blology, Harvard University. No. 187. 
adjoining tissues, which do not take the stain so readily. Balsam mounts were prepared from legs excised above the bases of the coxæ, fixed in Gilson's mercuro-nitric mixture and stained in either Ehrlich's acid hæmatoxylin (12 hours; then differentiated in 70 per cent. alcohol which had been slightly acidulated with hydrochloric acid, and dehydrated) or in alum-cochineal prepared according to Guyer's formula $^{2}$ (36 hours; then washed 20 minutes in several changes of water and dehydrated). Alum-cochineal was found to produce more delicate differentiation. Ehrlich's hæmatoxylin also gave good results, but its tendency to stain the material too deeply makes it inferior to the alum-cochineal for preparations in toto.

\section{Anatomical Considerations.}

Except in size and in some other minor differences, the three pairs of legs are apparently uniformly similar in their anatomical characters. In the absence therefore of any important structural modifications in any of the legs, no attempt is made in the present discussion to treat each of the three pairs separately.

The legs of aphids have two tarsal segments. In the case of Myzus persice the first segment (Plate IV, figures 1 and 3. C) is nearly one-fourth the length of the second (Plate IV, figures 1 and 3. c). The former is subtriangular from the lateral aspect, and is produced at the entoproximal ${ }^{3}$ margin into two subconical projections, as follows: (1) A longer one, which is subconfluent with the ental wall of the first tarsal segment (Plate IV, figures 3 and 5. $H$ ). This serves as the base for the insertion of a muscle which by its analogy in function to certain vertebrate muscles will be termed the extensor tarsi. ${ }^{4}$ (2) A smaller projection which branches from the former at the base and is directed entally with respect to the median line of the insect's body at an angle of about 90 degrees

\footnotetext{
${ }^{2}$ Guyer, M. F. 1917. Animal Micrology: 9. Chicago.

'In this and the paragraphs which follow, such terms as ental, ectal, etc., refer to the normal position of the leg with respect to the median line of the insect's body.

${ }^{4}$ In vertebrates, muscles are usually "arranged in antagonistic groups, the action of one being the opposite of its antagonist. Thus there are flexors to bend a limb, extensors to straighten it. . . ." Kingsley, J. S. 1917. Outlines of Comparative Anatomy of Vertebrates, second edition, revised: 134. Fhiladelphia.
} 
from the ental wall of the first tarsal segment (Plate IV, figures 3 and 5. I). This projection is connected at the apex and sides with the articular membrane (Plate IV, figures 3 and 5. $J$ ), and will be described presently. The ectal wall of the first tarsal segment is very short, and is broadly and deeply cleft at the proximal margin, forming an outer, round-top, subconical process, which fits rather snugly into a groove at the ectodistal margin of the tibia. This arrangement (Plate IV, figures 3 and $4 . G$ ) serves as the single hinge on which the tarsus moves, a condition contrasting with that of the adjoining segment, the tibia, since the latter, as pointed out by Woodworth (1908), moves on two hinges, situated on either side at its junction with the femur. At its ento- and lateroproximal sides, the first tarsal segment fits telescopically into the thickly chitinized distal wall of the tibia (Plate IV, figures 3 and 5). When the tarsus is extended, the intervening space is widest between the ental walls of the two segments and narrows down ectolaterally into the hinge. The proximal margin of the first tarsal segment apparently first snugly into the distal rim of the tibia when the former is flexed outward. The cuticular membrane connecting the proximal margin of the first tarsal segment with the distal margin of the tibia (Plate IV, figures 3 and $5 . J$ ) is a continuation of the cuticula of the leg and is very flexible owing to its being only thinly chitinized. It is widest entally and narrows ectolaterally into the hinge, remotely assuming when stretched a semilunar shape. The darker appearance of a small area immediately adjoining the tarsal projection, which has been referred to above, indicates a marked reinforcement in this region.

As has been mentioned in the foregoing paragraphs, a subconical projection at the entoproximal margin of the first tarsal segment serves as the base for the insertion of the extensor tarsi. This muscle, as far as I could make out, both in the fresh preparations and in balsam mounts, has its origin within the ectal wall of the tibia, near the proximal end of the latter (Plate IV, figures 1 and 2. $E)$. The body of the muscle is at the subdistal portion. This is a short, spindle-shaped mass, which is readily distinguishablo through the tibial wall in whole mounts (Plate IV, figure 3. $E$ ). 
A short tendon ${ }^{5}$ connects it with the projection of the tarsus. The tendon which connects this muscle with the subproximal end of the tibia is relatively much longer and narrowly subfusiform, abruptly expanding subconically toward the point of origin.

It is of considerable interest in connection with the musculature of the tarsi in aphids that the flexor is absent, its function having been taken over by another arrangement which will be describer presently. In the more primitive types of insects, as in the Blattidæ, two antagonistic sets of tarsal muscles are present. Miall and Denny (1886) found a flexor and a retractor moving the tarsi of Blatta orientalis Linn. The disappearance of the flexor tarsi in aphids must have been the result of degeneration consequent upon the more highly specialized habits of this insect.

The junction between the first and the second tarsal segments has not been satisfactorily studied in connection with the present work. It appears, however, that the two segments are more or less firmly connected by a narrow strip of articular membrane in such a way as to give the second tarsal segment only a very restricted amount of independent movement.

\section{Mechanism of Tarsal Movement.}

From an examination of the anatomical structure of the legs, as well as from direct study of their movements under the microscope, it is evident that movement in the tarsal region centers around the first tarsal segment. Apparently, the tension of the highly chitinized wall of the tibia has the tendency to stretch the articular membrane outward, and, since the single hinge is located at the ectal part of the articulation of the first tarsal segment with the tibia, where it offers resistance to the tension of the tibial wall, the tarsus is bent on this hinge at an angle of about 90 degrees. As the extensor tarsi contracts, an upward pull is applied on the entoproximal margin of the first tarsal segment. Pivoted on the

\footnotetext{
5 The term tendon, as used in the present paper, is applied to the more or less subcylindrical strand of tissue which connects the main body of the muscle with the inner wall of the insect's legs. As used by Woodworth (1908), the word apparently has a different significance. So far as I was able to make out from both his discussion and his figures, it is apparently meant by him to apply to the projection or elongation of the chitinous wall which serves as the muscular insertion.
} 
hinge, the tarsus is stretched medially and becomes extended so as to bring it at an approximately straight line with the tibia. Relaxation of the extensor removes the force which pulls the first tarsal segment at the place indicated, and the tension of the wall of the tibia brings the former back to its original position. It appears, therefore, that the wall of the tibia performs the function of the lost antagonistic muscle of the tarsi in aphids. The movement of the tarsi in either direction is limited by the articular membrane.

As has already been noted, the movement of the second tarsal segment is apparently very restricted, probably to the extent of merely giving the tarsus a certain amount of resiliency when the insect is walking. It has not been observed in the present work to flex to any marked extent either ectally or entally with respect to the first tarsal segment.

\section{SUMmary.}

In certain more primitive insects, two antagonistic muscles move the tarsus. In the aphids, one of these is lost through degeneration or atrophy, only the extensor remaining.

The function of the lost antagonistic muscle is taken over by the walls of the tibia, which, by its tension, has the tendency to flex the tarsus outward at an angle with the tibia.

The tarsus moves on a single hinge, located at the ectal portion of the articulation of the first tarsal segment with the tibia.

The articular membrane limits the movements of the tarsus in either direction.

The second tarsal segment is apparently very restricted in its movement, perhaps to the extent of only giving the tarsus a certain amount of resiliency when the insect is in motion.

\section{Literature Cited.}

Miall, L. C., and Denny, A. 1886. The Structure and Life History of the Cockroach (Periplaneta orientalis) : $7 \%$. Fig. 37. London and Leeds.

Woodworth, C. W. 1908. The Leg Tendons of Insects. The American Naturalist, 42: 452-456. 
Explanation of Plate IV.

Details of Legs of Myzus persicce Sulzer.

A. Femur.

B. Tibia.

C. First tarsal segment.

c. Second tarsal segment.

$D$. Ungues.

E. Extensor tarsi.

$F$. Hypodermis of tibia.

G. Hinge at articulation of first tarsal segment with tibia.

II. Subconical projection of entoproximal margin of first tarsal segment, serving as base for insertion of extensor tarsi.

I. Another projection of first tarsal segment, connecting with articular membrane.

$J$. Articular membrane connecting proximal margin of first tarsal segment with distal margin of tibia.

Figure 1. Lateral view of leg, showing relative positions of femur and more distal adjoining segments; muscle and hypodermis. $\times 85$.

Figure \&. Articulation and adjoining portions of femur and tibia, showing origin of extensor tarsi. J Jateral aspect. $\times 350$.

Figure 3. Distal portion of tibia and adjoining segments, showing insertion of extensor tarsi; articulation of first and second tarsal segments; hinge of first tarsal segment; and articular membrane. Lateral aspect. $\times 350$.

Figure 4. Ectolateral portion of leg adjoining articulation of first tarsal segment with tibia, showing hinge. Lateral aspect. $\times 675$.

Figure 5. Entolateral portion of leg adjoining articulation of first tarsal segment with tibia, showing articular membrane and the subconical projections at entoproximal margin of the first tarsal segment. Lateral aspect. $\times 675$. 


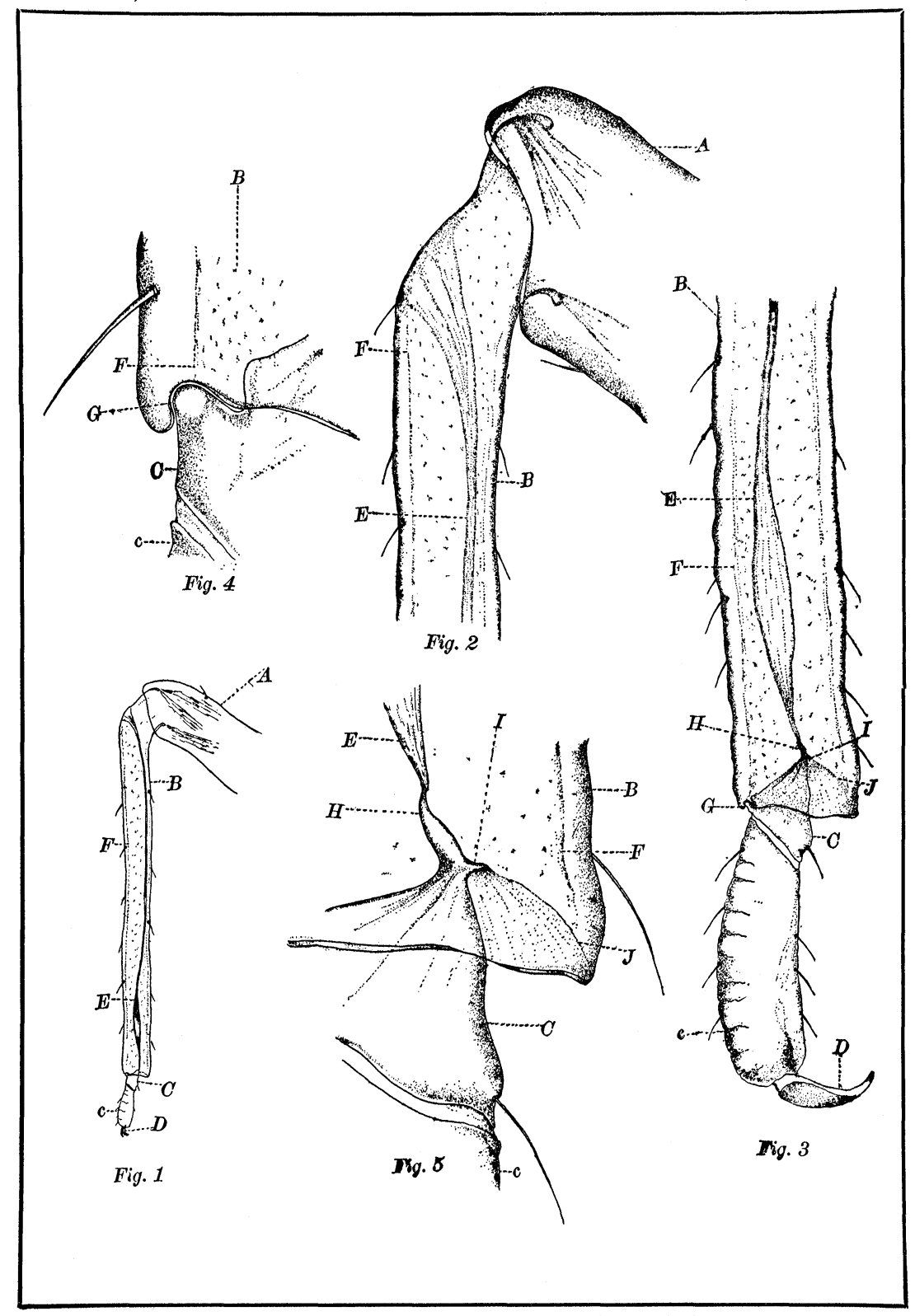

Uichanco-Musculature of Tarsi in Aphids. 

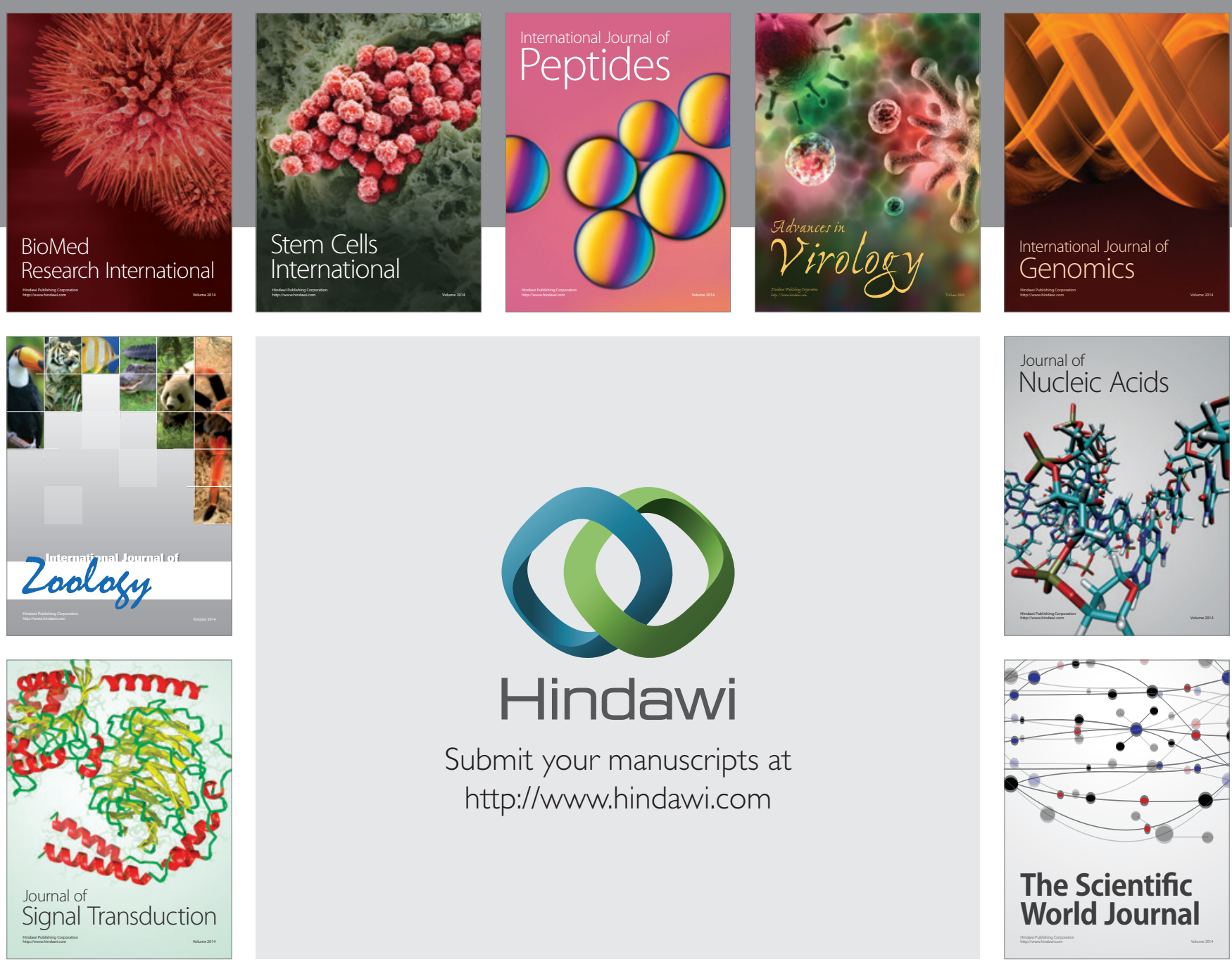

Submit your manuscripts at

http://www.hindawi.com
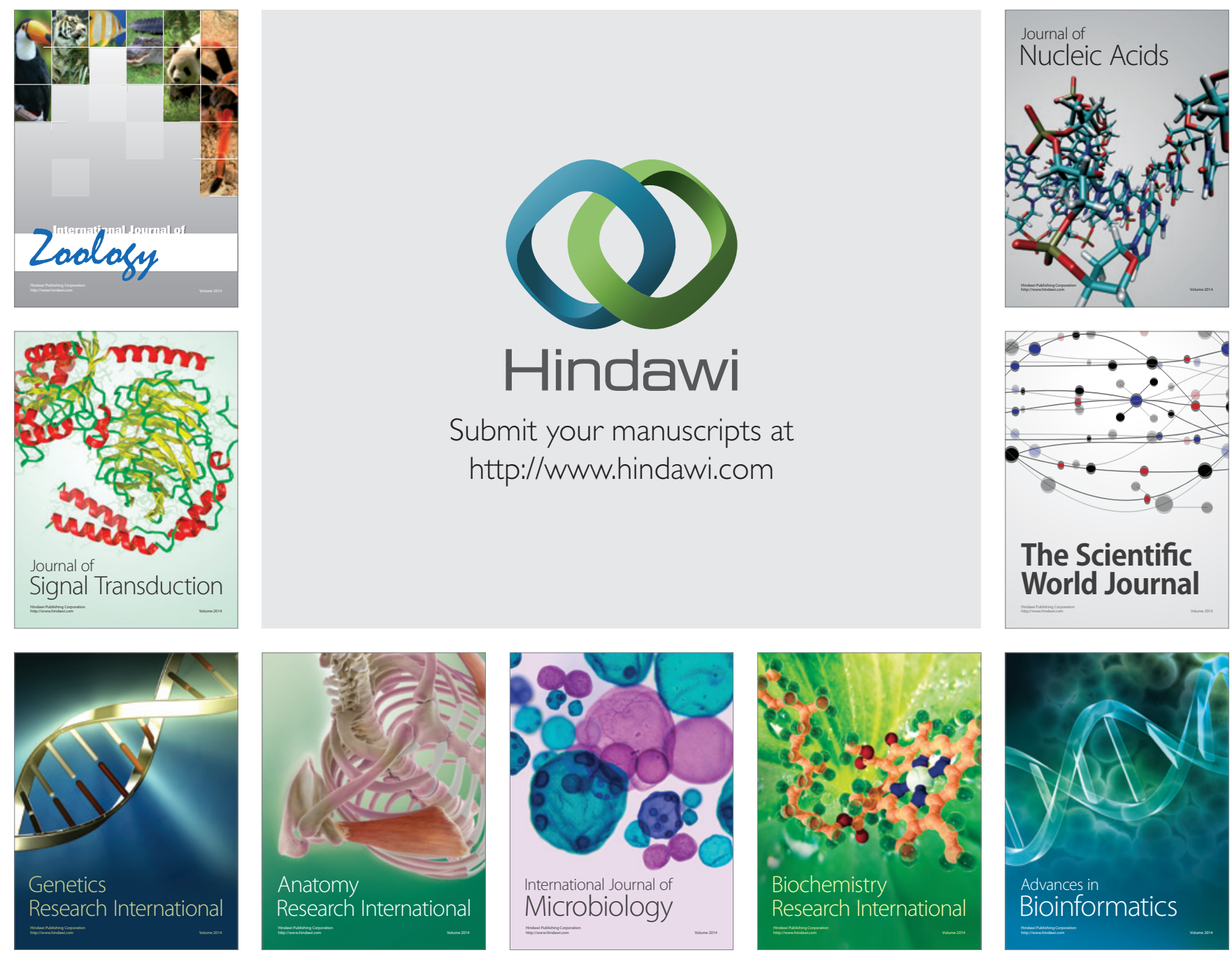

The Scientific World Journal
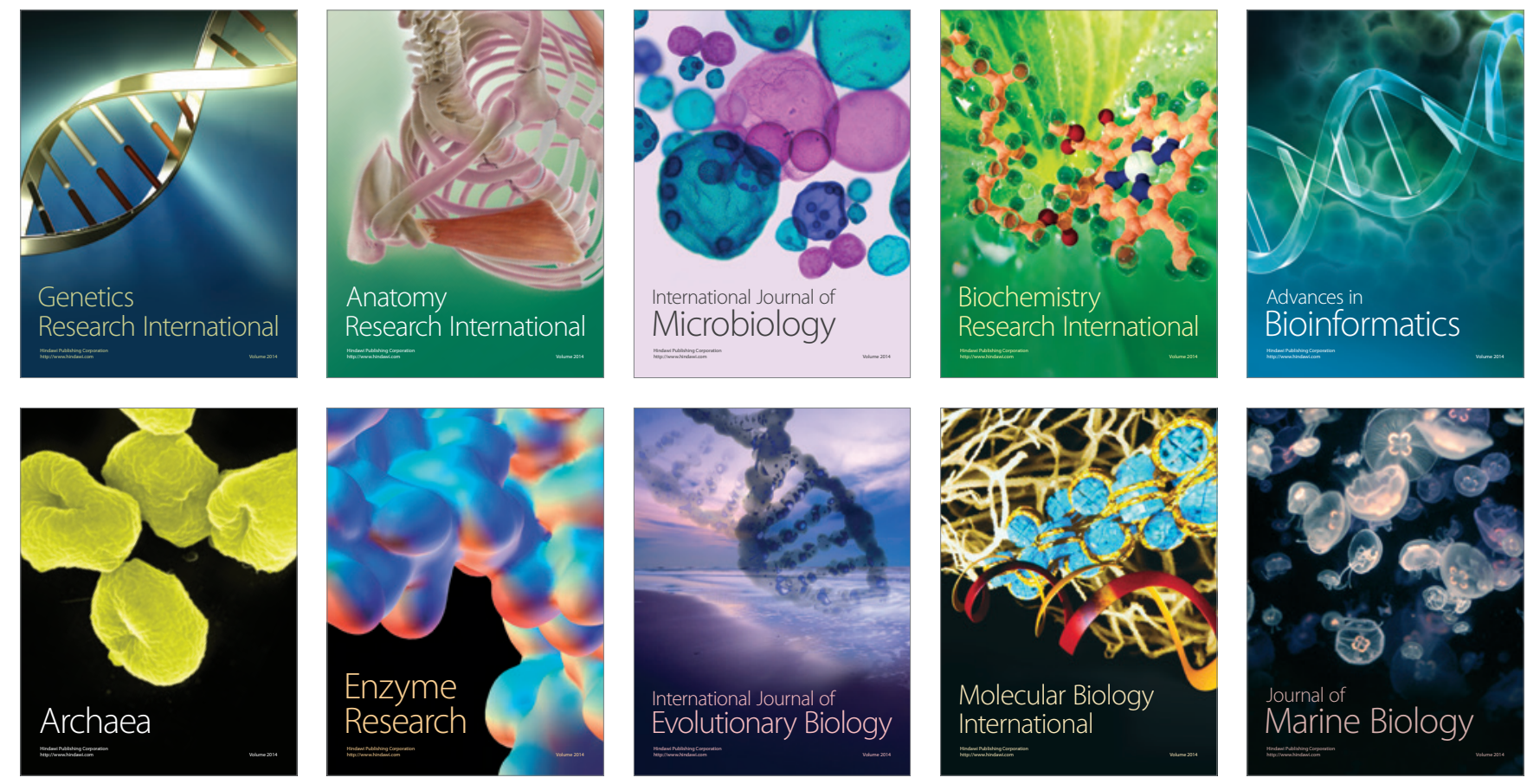